\title{
The Artistic Characteristics and Analysis of Singing of Mr. Shi Guangnan's Coloratura Vocal Works
}

\author{
Zhang Jing ${ }^{1}$ \\ ${ }^{1}$ Dalian Art College
}

Keywords: Mr. Shi Guangnan; Artistic characteristics; Analysis of singing; Coloratura vocal works

\begin{abstract}
Mr. Shi Guangnan has composed numerous works in his music career, and he has made especially remarkable achievements in the creation of coloratura vocal works. In this paper, several pieces of coloratura vocal works of Mr. Shi Guangnan are selected for analysis and research of the artistic characteristics and singing performance, and characteristics of the vocal works of Mr. Shi Guangnan are summarized.In this paper, six of Mr. Shi Guangnan's works are selected, which are Arises Above the Sea is a Bright Moon, Birds in the Woods are Singing, Crane, Lisai Love Song, Waiting Delightedly and Birds, My Friends, and their characteristics and singing are analyzed.
\end{abstract}

\section{Introduction to Mr. Shi Guangnan's coloratura}

Mr. Shi Guangnan represents the beginning of Chinese creation music, and he is one of the most well-known composers in New China, thereby having the good reputation of "Singer of the time". Mr. Shi Guangnan has remarkably outstanding achievements in creation and music. When he was in middle school, he had already written nearly 300 pieces of songs. When he was 7 years old, many of his songs had already been sung by people and won people's acknowledgement, so he is worthy of the honor of "people's musician".

Mr. Shi Guangnan has written so many works, and one of them has not drawn so much attention compared with other works, i.e., coloratura vocal works. Coloratura vocal works means adding rapid scale radical, tautophony, decomposed chord and trill into vocal music so that different tones can be presented in performance so that it can be more artistic. Major characteristics of coloratura vocal works are that it can be more artistic and rhythmic in simple melody. Coloratura vocal works have high demands for performers, and it is mainly represented in the following two aspects. Firstly, it requires good high pitch technique; secondly, the singer should control the breath and volume well.

Mr. Shi Guangnan has extremely high talents in the creation of coloratura music works. During the creation process, he often conducts deep research in folk music and traditional Chinese music to study the artistic expression of coloratura. During the 1970s and the 1980s, coloratura singing stimulation was the most popular, so Mr. Shi Guangnan conducted especially deep research in it, thereby creating some excellent coloratura vocal works. Among Mr. Shi Guangnan's music works, characteristics of time are very distinct, and the works have obvious national features. His works not only have high artistic value, but also are close to the public; they are very popular and "suit both refined and popular tastes", thereby wining people's great favor. Mr. Shi Guangnan's coloratura vocal works covers all aspects of films, operas, artistic songs, etc., and they are sung almost everywhere. Therefore, Mr. Shi Guangnan is of profound significance to the development of Chinese coloratura art.

\section{Analysis of artistic characteristics of Mr. Shi Guangnan's coloratura vocal works}

Music is a kind of performance art. It is favored by numerous people and is the most essential demand in people's heart, which is the significance of the existence of music. Characteristics of time are very distinct in Mr. Shi Guangnan's music, and the works have bright national features. His works not only have high artistic value, but also are close to people; they are very popular and "suit 
both refined and popular tastes", thereby wining people's great favor. Mr. Shi Guangnan is familiar with both Western music and folk music. He combined them and formed his unique artistic values. Some of his music works involve comprehensive themes and have vitality and time characteristics.

Mr. Shi Guangnan's works not only have lively rhythm but also national features. When talking about Chopin, most of us would think of piano music; when talking about Beethoven, we would think of music saint; when talking about Haydn, we would think of symphony. Every musician has his unique side, and all these famous musicians have the same thing, i.e., their music is related to the nation, just like Beethoven's Symphony of Fate. Mr. Shi Guangnan's works also have such characteristics. He combined Western music with Chinese music and added Chinese national instruments into it, thereby forming music with distinct national features.

Once, a musician proposed that "melody is the essence of music", and outstanding musicians in the world usually have a generality, i.e., they control melody strictly. Mr. Shi Guangnan's works have distinct individuality and vivid characteristics in the representation of coloratura vocal works; in coloratura vocal works, rapid scale radical, tautophony, decomposed chord and trill are added into vocal melody to represent different tones in performance and make the music more artistic. Mr. Shi Guangnan's works usually have graceful melody, lively rhythm and distinct characteristics.

During piano accompaniment, Mr. Shi Guangnan pays much attention to the arrangement of harmony so that they can be characteristic in the whole rhythm; meanwhile, he takes full consideration of the artistic conception and style of the song. The acoustics of the harmony of Mr. Shi Guangnan's works is usually very rich, and the accompaniment suits the melody. Different rhythm and expression ways match with different artistic conceptions so that audiences can be fully integrated into the artistic conception and be personally on the scene.

\section{Analysis of works}

\section{(1) Lisai Flying Song}

This song is a classic piece of coloratura vocal works, and it mainly expresses the beautiful yearning of the Li nationality. The song is divided into two parts. Part One is the main melody which is used to express feelings. Rhythm of Part Two is accelerated a little. In this way, Li people's praises and yearning for beautiful life is expressed. Mr. Shi Guangnan integrated traditional Li music and Western music into this song, thereby creating a song with bright features. This song is of high artistic value, and the singing is of huge difficulty. Meanwhile, it is an epitome of Li people's life. The coming of this song is of great importance to the protection of traditional Li music.

This song is also one of Mr. Shi Guangnan's classic works. It describes the story that the hero and heroine began their happy life after regaining love. The beginning of this work describes that the heroine is waiting for the coming of the hero, and it fully expresses their love. At that time, the heroine is immersed in happiness, and she is full of expectation and delight for their future life. Therefore, the rhythm of this work is lively, and it is a piece of allegretto song. The emotions are full and the lyricism is strong.

This song is a piece of film music work. The film has included several pieces of Mr. Shi Guangnan's coloratura vocal works, which are Sprays of Love and Birds in the Woods are Singing. To film music, the most important point is to reflect the theme of the film and the main content. These two songs play critical roles in the expression of the theme and the protagonists' ideas. When the protagonist is singing Birds in the Woods are Singing, this point is reflected profoundly, which promotes the development of the plots well.

The creation background of this work is the 1970s. At that time, China was establishing diplomatic relations with Japan, and the relationship between China and Japan was not so intense. There began to be communication between the two countries in politics, culture, economy and other aspects. As a representative person of Chinese artistic culture at that time, Mr. Shi Guangnan often attended such Sino-Japan cultural activities. Based on this time background, Mr. Shi Guangnan created this Crane. The melody of this song is lively and full of emotions. By praising cranes, the composer expresses his yearning and pursuit for peace as well as the wish that Sino-Japan friendship can last forever. 
This song is one of the most classic works of Mr. Shi Guangnan. Many people believe this is a song with the highest artistic values in all his coloratura vocal works. This song also appears in competitions frequently. It was composed in the 1980s, when the concept of ecological environment protection was proposed. Based on this background, this song was composed. This song is a coloratura soprano, since woman's voice can better present birds' chirp, and the artistic conception of this song can be better presented. However, since the performance difficulty of this song is too high, it was not presented to audiences until 2002.

Song of A Mountain Ghost was composed in the 1980s for the drama Quyuan. The drama was adapted, so the music images of this song are very lively. This song is mainly to express the love of the goddess of Mountain Wu. It describes the heroine's pursuit for love as well as her psychological feelings. The melody is long, fresh, lyric and romantic, and it is a piece of God-given work.

\section{Analysis of the singing of Mr. Shi Guangnan's coloratura vocal works}

It is a kind of tone that relies on major tones, and it can be divided into short appoggiatura and long appoggiatura. When short appoggiatura is being performed, it does not take up theoretical duration; during actual performance, it would take up the duration of major tones. However, it still depends on the work. Long appoggiatura is usually expressed by small note which is no larger than crotchet, and it should be analyzed in specific work. In Mr. Shi Guangnan's work Birds, My Friends, it is adopted. In the last sentence, seven appoggiaturas are adopted to express birds' chirp so that audiences can be personally on the scene.

There is no difference between Chinese and Western singing way of staccatos. There are three ways in the expression of notes, which are arc, dot and triangle. Staccato requires the singer to have good voice state. The most basic expression of coloratura techniques is staccato, and it exists in many of Mr. Shi Guangnan's works. For example, in the work Waiting Delightedly, during the singing, the heroine's delighted feelings when waiting for the hero should be presented.

It is a tone formed by rapid alternation of major tone and adjacent tones, and it is usually expressed by numbers of trill. If there is appoggiatura before the trill, the performance should start from the appoggiatura, but it still depends on specific situations. Techniques of trills appear in many of Mr. Shi Guangnan's works, such as Birds, My Friends, Waiting Delightedly, etc. During singing, there is a little difference between trills and other tones. It is produced from vibration of the throat instead of the chest.

Rapid scale is the most important part in coloratura vocal works, and the difficulty is the highest because of its complexity. Therefore, scales of this type of coloratura works are magnificent and charming. This point is presented profoundly in many of Mr. Shi Guangnan's coloratura vocal works. For example, in the song Spray of Love, this point is cited. During the performance, the singing is very difficult; it requires the singer to grasp the scale rhythm well as well as stable breath. To perform the song flexibly, the song needs to be sung coherently instead of dividing it into sections. The song must be sung coherently.

\section{Conclusion}

Mr. Shi Guangnan's works belongs to the era as well as the whole nation. On the basis of traditional Chinese music, he integrated Chinese music into it; meanwhile, his works penetrate deep into the public; they have his unique charm as well as strong lyric nature. He processes the rhythm and the melody smoothly. The works are branded with time, and his works are worthy of inheritance.

\section{References}

[1] Cai Li. Analysis of the Singing of Coloratura Vocal Works of Chinese Style_—On the Control of Singing in Folium Cycadis Revolutae of a Thousand Years Bloom [J]. Journal of Mianyang Teachers' College, 2010,(12). 
[2] Wang Shanming. Creation and Singing of Shang Deyi's "Coloratura" Vocal Works in the late 20th Century [J]. Northern Music, 2014,(2).

[3] WeiYijun. Application of Shang Deyi's Coloratura Art Songs in the Teaching of Vocal Music. People's Music (The Critical Edition), 2008, (4).

[4] Cao Ke. Case Study of the Singing of Vocal Works in Baroque Period——With Bartoli as Research Object [D]. Central China Normal University, 2016.

[5] Wang Xiuhong. Analysis of the Tragedy of Handel's Vocal Works-With Aria Let Me Cry as an Example [J]. Music View, 2014,(11).

[6] Xu Zheng. On How to Sing Birds, My Friends from the Perspective of Music Performance Aesthetics [J]. Popular Literature, 2014 (15). 